\title{
A Review of Multi-Echelon Inventory Control in Supply Chain
}

\author{
Jiao Sun \\ School of Management, Jinan University, Guangzhou, China \\ Email:mse_sunj@126.com
}

How to cite this paper: Sun, J. (2020) A Review of Multi-Echelon Inventory Control in Supply Chain. Open Journal of Business and Management, 8, 881-891.

https://doi.org/10.4236/ojbm.2020.82054

Received: March 4, 2020

Accepted: March 22, 2020

Published: March 25, 2020

Copyright $\odot 2020$ by author(s) and Scientific Research Publishing Inc. This work is licensed under the Creative Commons Attribution International License (CC BY 4.0).

http://creativecommons.org/licenses/by/4.0/

\begin{abstract}
Multi-echelon inventory control is the core issue in supply chain. Firstly, we summarize the current research status of multi-echelon inventory control by using Citispace which is the scientific knowledge map visualization analysis software. Second, two strategies of multi-echelon inventory optimization and control are analyzed. Then, multi-echelon inventory control problem is divided into three strategies, namely multi-echelon multi-node inventory control strategy, multi-echelon single-node inventory control strategy, and transshipment-based multi-echelon multi-node inventory control strategy, and comparative analysis. Finally, we point out future development trend.
\end{abstract}

\section{Keywords}

Multi-Echelon Inventory Control, Control Strategy, Development Trend

\section{Introduction}

For enterprise, inventory is a double-edged sword, which can alleviate the random factors and emergency demand in production and supply, but also occupy substantial working capital and reduce the operational efficiency of the enterprise. In each stage of the supply chain, certain inventory needs to be guaranteed to ensure the continuity of production and inventory. Therefore, effective inventory control and management can maintain the inventory of each node in the supply chain at a reasonable level which has important practical significance. The availability of inventory has a very important impact on business operations. With the development of supply chain management, it is necessary to study multi-echelon inventory control, analyze multiple strategies for multi-echelon inventory optimization and control, and face different multi-echelon inventory control issues adopting different strategies to make supply chain management develop better. 
The multi-echelon inventory control problem is mainly to study the inventory problem of each inventory point in the supply chain. The main decision goal is to make the efficiency of the entire supply chain optimal and the cost of the supply chain to be the lowest. However, there are many uncertain factors in multi-echelon inventory management, such as random changes in customer demand, uncertain order lead times, etc., and the number of echelons and nodes involved in multi-echelon inventory control ranges, which makes inventory control management and coordination difficult.

Therefore, we analyze the volume of multi-echelon inventory research literature published in academic journals at home and abroad from 2009 to 2019. Through the analysis of the literature, we reveal the research status, problems and main results of multi-echelon inventory control in the past decade at home and abroad. It can be used as a reference for researchers and users of multi-echelon inventory control to promote the research of multi-echelon inventory control.

\section{Research Methods}

\subsection{Research Methods}

We use a combination of quantitative and qualitative research, and uses the information visualization statistics software Citispace developed by Dr. Chaomei Chen from Drexel University in the United States. From the keywords and research content of the paper, we classified statistics and analysis of multi-echelon inventory control literature published in academic journals at home and abroad in the past decade. CiteSpace is a scientific knowledge atlas citation analysis software. It embeds the CNKI (ReWork) format conversion program compiled by Dr. Liu Shengbo of Dalian University of Technology. It can convert the collected data into a processable format, which can help researchers determine the evolution of the subject knowledge domain Visual analysis with research hotspots [1] [2].

\subsection{Research Note}

In this paper, we use the subject search method and the coverage of search results is higher than that of title search and keyword search, but it may not cover all the literature on multi-echelon inventory control. In addition, considering maneuverability, our research has no statistical academic thesis.

Although our research strives to be complete and accurate in the statistics of the data, due to the different requirements of authors and other information in different journals, some of the original document information is incomplete (such as the lack of author names, unknown author units, etc.). When the information is imported into Citispace, we leave the missing information blank. In addition, due to the limitation of the researcher's own knowledge structure, it will inevitably cause artificial classification bias, which may cause certain errors in the classification of the research content. On the whole, this study adheres to 
the principle of fairness and fairness, and has high statistical significance. The overall situation presented after the study of statistics is scientific and credible, and has scientific application value.

\section{Multi-Echelon Inventory Control Literature Collection}

We mainly use CNKI and WEB OF SCIENCE to search the literature. CNKI uses "multi-level inventory control" keywords for topic search. The search results are relatively small. Citespace is not used for data processing. Figure 1 shows CNKI from the index analysis results of "multi-level inventory control". It comes from CNKI. The blue line indicates scholarly attention to multi-echelon inventory control. The red line refers to the sequential growth rate of scholarly attention to multi-echelon inventory control. We can see that multi-echelon inventory control has lower attention, there is less literature in ten years. There is a significant decrease in the number of literature compared with 2011.

In the WEB OF SCIENCE, the keywords "multi-echelon inventory control", "multi-stage inventory control", and "multi-level inventory control" were used for topic search. The searched documents were analyzed using Citespace for data analysis. They are showing in Figure 2 and Figure 3.

From the above analysis, we can see that the focus research in chain is mainly on multi-echelon inventory models, optimization, simulation, strategy, and systems. For the research of multi-echelon inventory, it was first started by the level inventory proposed by Clark A. and Scarf H. [3], and then gradually started from the two echelons inventory, and then to the three echelons to more levels later. For example, Minner S. et al. (2003) studies used a cycle inventory strategy to optimize secondary inventory to achieve appropriate replenishment, they studied the impact of manufacturing flexibility on inventory investment in a distribution network composed of a central warehouse and several local inventory points [4]. Moinzadeh K. (2002) proposed the use of rapid exchange of information to achieve multi-echelon inventory cost control based on the $(\mathrm{Q}, \mathrm{R})$ strategy [5]. Gerard P. Cachon and Marshall Fisher (2004) studied the role of information

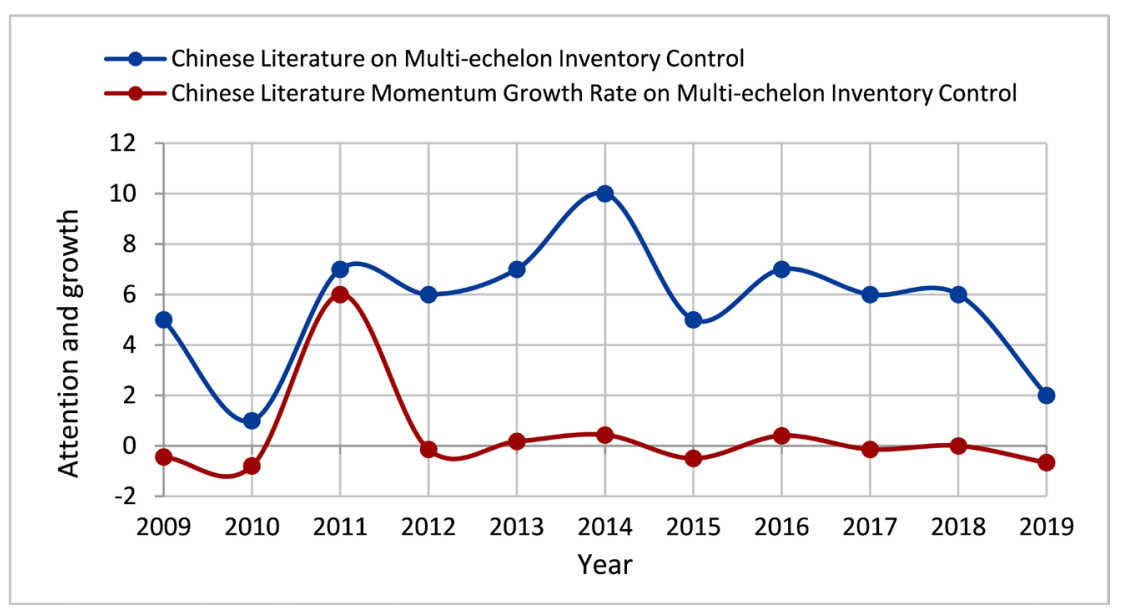

Figure 1. Index analysis results of multi-echelon inventory control. 


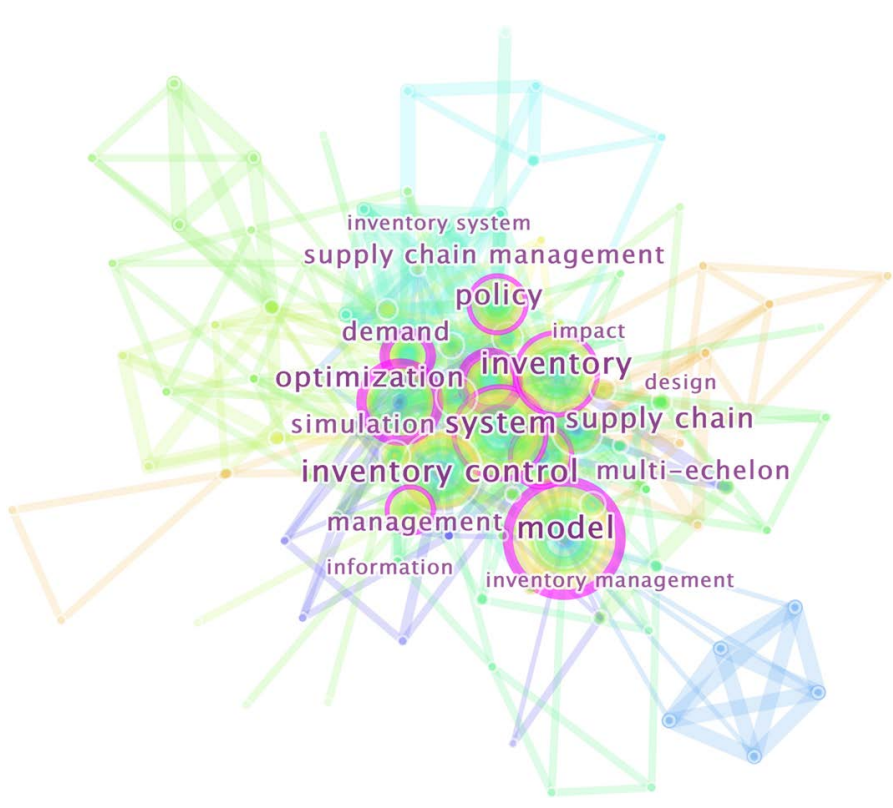

Figure 2. Cluster analysis of WEB OF SCIENCE search keywords.

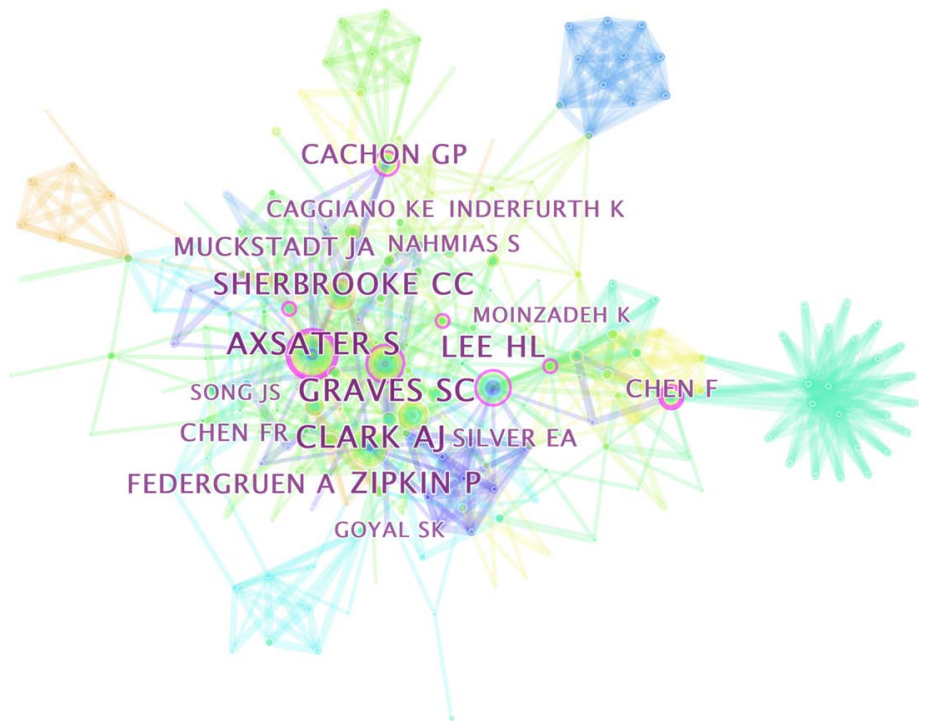

Figure 3. Co-introduction of the search keywords for WEB OF SCIENCE.

sharing cost simplification of the inventory model [6]. In recent years, Hu Xiaojian et al. (2016) optimized the three-tier inventory model of service spare parts [7]. Chen Qian (2017) studied the multi-echelon inventory control problem in the operation of the mesh supply chain, built a multi-echelon inventory control model for the supply chain, and tested the validity of the model [8]. Alawneh et al. (2018) studied multi-echelon inventory management under stochastic demand [9]. Jalili Marand A et al. (2019) study the joint inventory control and pricing decision of service inventory system [10].

\section{Multi-Echelon Inventory Optimization and Control Strategy}

We find the research on supply chain multi-echelon inventory control methods 
mostly focuses on decentralized and centralized strategies. These two strategies are widely used in supply chain multi-echelon inventory control due to their own characteristics and scope of application.

\subsection{Decentralized Strategies}

The decentralization strategy refers to the operation of each inventory point in the supply chain independently, each adopting its own inventory strategy [11]. Each node determines the order point and order quantity according to its own ordering strategy and local inventory optimization strategy. It has also named distributed control strategy. Early research on multi-echelon inventory control strategies mainly focused on decentralized inventory control strategies, such as Harris's famous economic order lot model in 1915 and Ramasesh's optimal production lot model in 1990. These studies mainly discuss the optimal inventory control strategies of each node in the supply chain.

The decentralized strategy uses multiple cost settlement centers. Each center implements control according to actual needs. It is relatively difficult to manage. It requires a high degree of information sharing and effective information integration. The decentralized strategy is only a partial optimization and cannot ensure that inventory activities are optimal throughout the supply chain [12].

\subsection{Centralized Strategies}

The centralized strategy refers to the inventory control center placed on the core enterprises of the supply chain. The core enterprises are responsible for data integration and coordination functions, and control the overall inventory of the supply chain to coordinate the completion of inventory activities upstream and downstream of the supply chain [13].

In 1960, Clark and Scarf began to study multi-echelon inventory problem in the supply chain, and proposed the concept of "echelon inventory" [3]. A deterministic ordering batch multi-echelon inventory model considering the out-of-stock penalty is constructed, and its optimal inventory control strategy is proved to be the maximum order level. This makes the research focus of supply chain inventory control strategies begin to shift from decentralized strategies to centralized strategies. Subsequently, many scholars began to study the centralized multi-echelon inventory management in different supply chain environments. For example, Jain and Raghavan (2009) proposed a multi-echelon inventory model, which consists of a manufacturer, a warehouse, and a retailer. They determine the optimal inventory level to minimize the total expected cost [14]. You and Grossmann (2010) used a guaranteed service approach to design a multi-echelon supply chain with an uncertain inventory system. This multi-echelon supply chain includes factories, distribution centers, and customer demand areas [15]. Yu (2010) developed a three-tier supply chain (a retailer, a distributor, and a supplier) inventory model for perishable products using heuristics to minimize total costs [16]. Cannella et al. (2011) proposed an inventory model and showed 
how to modify the replenishment rules of a multi-echelon supply chain [17]. Wang et al. (2011) proposed an optimal inventory strategy for degraded items in a three-tier supply chain (a producer, a distributor, and a retailer). They examined the impact of different deterioration rates on inventory policies [18]. Guo and $\mathrm{Li}$ (2014) proposed a multi-echelon inventory system to study integrated supplier selection and inventory control in supply chain management [19]. Ross et al. (2016) proposed a multi-echelon location inventory model for industries driven by donation demand to minimize the total annual cost, and solved the model using the Lagrangian relaxation method [20].

All functional links of the centralized inventory control strategy can be integrated and coordinated, which not only reduces the overall operating costs of the supply chain, but also improves the customer service level in supply chain. However, the strategy aims at the lowest total cost of the supply chain and does not consider other optimization goals. As the number of nodes and stages increases, it becomes more difficult to coordinate the relationship between nodes and adjust inventory activities.

\section{Three Multi-Echelon Inventory Control Strategies}

Model is one of the focuses of the research on multi-echelon inventory control. According to the complexity of the model, the research of multi-echelon inventory control strategy is divided into three categories: multi-echelon multi-node inventory control strategy, multi-echelon single-node inventory control strategy, and multi-echelon multi-node inventory control strategy considering transfer.

\subsection{Multi-Echelon Multi-Node Inventory Control Strategy}

The inventory control problem is a tactical level problem, and in the actual operation of the enterprise, not only the tactical level problem is considered. Therefore, at present, when many scholars do research on multi-echelon and multi-node inventory control strategies, in order to avoid local optimization, they will combine the strategic level of facility location or operation level of path planning problem for global optimization. For example, Tancrez (2012) studied the location-inventory problem of a three-tier supply chain network [21]. Hamedani et al. (2013) used a multi-objective mixed integer non-linear programming modeling approach to research on the location-inventory problem of the three-tier supply chain network under the uncertain environment [22]. Of course, some scholars only research on multi-echelon inventory management problems through random or fuzzy programming. For example, Gumus and Guneri (2009) provide a multi-echelon multi-node inventory management framework under uncertainty or fuzzy environment, they give an application example of a three-tier, three-branch supply chain network [23]. Ross et al. (2016) studied the location-inventory problem of a three-tier supply chain in the demand-driven industry of donation [20]. Vahdani et al. (2017) built a three-level supply chain location-inventory model for a hybrid integer nonlinear 
programming optimization model with minimum supply chain costs under the assumption that the retailer's needs are relevant and allow inventory shortages [24].

\subsection{Multi-Echelon Single-Node Inventory Control Strategy}

The earliest studies, such as Clark and Scarf, involved a multi-echelon single-node inventory control strategy, which is characterized by each level having only one large warehouse. The modeling method adopted by Clark and Scarf (1960) is dynamic programming, which studies the problem of a fixed period and fixed order lot [3]. Then, for example, Bodt and Graves of MIT (2011) studied the multi-echelon single-node inventory problem under multi-cycle Poisson demand, and each node adopted a continuous inventory (R, Q) inventory control strategy [25]. Wen Q et al. (2013) reduced the cost of spare parts by optimizing the inventory management model [26]. Sven Axsater's research [27] [28] compared the advantages and disadvantages of multi-echelon joint inventory management and multi-echelon distributed inventory management.

\subsection{Multi-Echelon Multi-Node Inventory Control Strategy Considering Transfer}

In the actual operation of the enterprise, when the inventory is facing shortage, the enterprise has two measures: one is to wait for the replenishment of the upper layer, and the other is to meet the demand through transshipment. The transfer can be divided into the same-level transshipment same-level transshipment and the upper-level transshipment. The earliest Colin Paterson (2011) reviewed the inventory model of same-level transshipment. Among them, the same-level transshipment can be divided into completely same-level transshipment and partial same-level transshipment [29]. Liao Y (2014) studied the advantages and disadvantages of two strategies of emergency ordering and horizontal transfer when the retail industry is out of stock. The analysis shows that when the customer needs more or more customers go to another store, it is better to use the emergency ordering strategy. At the same time, the strategy of using emergency ordering when horizontal transfer cannot meet the demand is also studied [30].

Dan et al. (2017) studied the preventive transshipment and ordering decision of perishable products during multiple sales stages [31].

A multi-echelon, multi-node inventory control strategy that considers transshipment can alleviate the losses caused by the shortage of each node to a certain extent, while reducing the safety inventory of each node, thereby saving a lot of costs. However, regardless of the inventory model considering the same-level transshipment or the upper-level transshipment, most of the current researches are still only two-tier inventory models, while the multi-tier inventory models are relatively few. And the modeling methods adopted are mostly deterministic modeling or directly solved by simulation, and relatively few random models. 


\subsection{Comparison}

The research on multi-echelon inventory control strategies is mainly concentrated in the above three categories, but among these three types, the simplest model establishment is multi-echelon single-node multi-echelon inventory control strategies, followed by multi-echelon multi-node inventory control strategies and the most complicated is the multi-echelon and multi-node inventory control strategy considering transfer. However, in practical applications, the most likely to be applied is a multi-echelon multi-node inventory control strategy, and relatively few cases are considered for transshipment, while multi-echelon single nodes have been stuck in the theoretical aspect.

In addition to cost competition, competition between supply chains also has time competition. Multi-echelon single-node inventory control strategies such as joint inventory reduce the cost of inventory. The information flow puts forward higher requirements. Although the multi-echelon multi-node can meet the time demand, it has increased the cost of the supply chain. However, the multi-echelon multi-node inventory control strategy considering transfers takes into account both time and cost. By considering the transfer, the random fluctuations in demand of each node are mitigated, and the safety inventory of each node is reduced which can reduce the cost of the supply chain. Thus, our research has great research significance. However, in the construction of a multi-echelon multi-node inventory problem model that considers transshipment, because there are many nodes and each node is related to each other (replenishment and transshipment at the same level, transshipment at the same level), and uncertain environments such as stochastic demand, random Lead time, etc., will make the model relatively complicated, so there are relatively few current studies.

\section{Conclusions}

Based on the above analysis, multi-echelon inventory control is based on current status studies and is not combined with the current background. Most literature on multi-echelon inventory control is based on the improvement of existing models and there is no new model. Multi-echelon inventory problem with three or more levels is more complicated, and there are few research results. And there is little research on multi-echelon inventory control. Then, the research on multi-echelon inventory control under dual channels or new retail, the research on multi-echelon inventory control models, the research on multi-echelon inventory control problems of three levels and above, and the multi-echelon inventory control problems of multi-products are all that will continue to be the future development trend.

Therefore, this paper analyzes the literature on multi-echelon inventory control from 2009 to 2019, points out the current research status of multi-echelon inventory control, and introduces two strategies for multi-echelon inventory optimization and control: decentralized and centralized strategies. Based on the complexity of the model, the research on multi-echelon inventory control strate- 
gies is classified and compared. Finally, the future research trends of multi-echelon inventory control are pointed out, which provides a basis for the follow-up scholars' research on multi-echelon inventory control.

Because this paper only classifies the research of multi-echelon inventory control strategies based on the complexity of the model, it does not consider other dimensions, such as optimization methods, simulation strategies, etc. In the future, there will be more aspects of multi-echelon inventory control strategies.

\section{Conflicts of Interest}

The author declares no conflicts of interest regarding the publication of this paper.

\section{References}

[1] Chen, C. and Leydesdorff, L. (2014) Patterns of Connections and Movements in Dual-Map Overlays: A New Method of Publication Portfolio Analysis. Journal of the Association for Information Science \& Technology, 65, 334-351. https://doi.org/10.1002/asi.22968

[2] Cas, J. (2017) Science Mapping: A Systematic Review of the Literature. Journal of Data and Information Science, 2, 1-40. https://doi.org/10.1515/jdis-2017-0006

[3] Clark, A.J. and Scarf, H. (1960) Optimal Policies for a Multi-Echelon Inventory Problem. Management Science, 6, 475-490. https://doi.org/10.1287/mnsc.6.4.475

[4] Minner, S., Diks, E.B. and Kok, A.G.D. (2003) A Two-Echelon Inventory System with Supply Lead Time Flexibility. AIIE Transactions, 35, 117-129. https://doi.org/10.1080/07408170304383

[5] Moinzadeh, K. (2002) A Multi-Echelon Inventory System with Information Exchange. Management Science, 48, 414-426. https://doi.org/10.1287/mnsc.48.3.414.7730

[6] Cachon, G.P. and Fisher, M. (2000) Supply Chain Inventory Management and the Value of Shared Information. Management Science, 46, 1032-1048. https://doi.org/10.1287/mnsc.46.8.1032.12029

[7] Hu, X.J. and Liu, X.L. (2016) Optimize Three-Echelon Inventory Model of Large Equipment Repairable Spare Parts. Logistics Engineering and Management, 38, $121-124+118$.

[8] Chen, Q. (2017) Study on Multi-Echelon Inventory Control in Netted Supply Chain in Stochastic Demand Environment. Logistics Technology, 36, 153-156.

[9] Alawneh, F. and Zhang, G. (2018) Dual-Channel Warehouse and Inventory Management with Stochastic Demand. Transportation Research Part E Logistics and Transportation Review, 112, 84-106. https://doi.org/10.1016/j.tre.2017.12.012

[10] Jalili Marand, A., Li, H. and Thorstenson, A. (2019) Joint Inventory Control and Pricing in a Service-Inventory System. International Journal of Production Economics, 209, 78-91. https://doi.org/10.1016/j.ijpe.2017.07.008

[11] Moorthy, K.S. (1988) Strategic Decentralization in Channels. Marketing Science, 7, 335-355. https://doi.org/10.1287/mksc.7.4.335

[12] Chen, S.Y. (2007) Inventory Control Policy in Supply Chain. Xiamen University, Xiamen.

[13] Cheng, M. (2014) The Research and Application of Supply Chain Inventory Control Problems. Lanzhou Jiaotong University, Lanzhou. 
[14] Jain, S. and Raghavan, N.R.S. (2009) A Queuing Approach for Inventory Planning with Batch Ordering in Multi-Echelon Supply Chains. Central European Journal of Operations Research, 17, 95-110. https://doi.org/10.1007/s10100-008-0077-8

[15] You, F. and Grossmann, I.E. (2010) Integrated Multi-Echelon Supply Chain Design with Inventories under Uncertainty: MINLP Models, Computational Strategies. AIChE Journal, 56, 419-440. https://doi.org/10.1002/aic.12010

[16] Yu, J.C.P. (2010) Optimal Deteriorating Items Inventory Model with a Three-Echelon Supply Chain Strategic Alliance. Asia-Pacific Journal of Operational Research, 27, Article ID: 1000295. https://doi.org/10.1142/S0217595910002958

[17] Framinan, J.M. (2011) Inventory Policies and Information Sharing in Multi-Echelon Supply Chains. Production Planning \& Control, 22, 649-659.

https://doi.org/10.1080/09537287.2010.512276

[18] Wang, K.J., Lin, Y.S. and Yu, J.C.P. (2011) Optimizing Inventory Policy for Products with Time-Sensitive Deteriorating Rates in a Multi-Echelon Supply Chain. International Journal of Production Economics, 130, 66-76. https://doi.org/10.1016/j.ijpe.2010.11.009

[19] Guo, C. and Li, X. (2014) A Multi-Echelon Inventory System with Supplier Selection and Order Allocation under Stochastic Demand. International Journal of Production Economics, 151, 37-47. https://doi.org/10.1016/j.ijpe.2014.01.017

[20] Ross, A., Khajehnezhad, M., Otieno, W., et al. (2016) Integrated Location-Inventory Modelling under Forward and Reverse Product Flows in the Used Merchandise Retail Sector: A Multi-Echelon Formulation. European Journal of Operational Research, 259, 664-676. https://doi.org/10.1016/j.ejor.2016.10.036

[21] Tancrez, J.S., Lange, J.C. and Semal, P. (2012) A Location-Inventory Model for Large Three-Level Supply Chains. Transportation Research Part E Logistics \& Transportation Review, 48, 485-502. https://doi.org/10.1016/j.tre.2011.10.005

[22] Hamedani, S.G., Jabalameli, M.S. and Bozorgiamiri, A. (2013) A Location-Inventory Model for Distribution Centers in a Three-Level Supply Chain under Uncertainty. International Journal of Industrial Engineering Computations, 4, 93-110. https://doi.org/10.5267/j.ijiec.2012.10.004

[23] Gumus, A.T. and Guneri, A.F. (2009) A Multi-Echelon Inventory Management Framework for Stochastic and Fuzzy Supply Chains. Expert Systems with Applications, 36, 5565-5575. https://doi.org/10.1016/j.eswa.2008.06.082

[24] Vahdani, B., Soltani, M., Yazdani, M., et al. (2017) A Three Level Joint Location-Inventory Problem with Correlated Demand, Shortages and Periodic Review System: Robust Meta-Heuristics. Computers \& Industrial Engineering, 109, 113-129. https://doi.org/10.1016/j.cie.2017.04.041

[25] Bodt, M.A.D. and Graves, S.C. (2011) Continuous Review Policies for a Multi-Echelon Inventory Problem with Stochastic Demand. Management Science, 31, 1286-1299. https://doi.org/10.1287/mnsc.31.10.1286

[26] Wen, Q., Fan, S., Xiong, T., et al. (2013) Cutter Suction Dredger Spare Parts Joint Inventory Management Research. Applied Mechanics and Materials. Proceedings of 2013 4th International Conference on Information Technology for Manufacturing Systems, Auckland, 28 August 2013, 889-893.

[27] Axsäter, S. and Zhang, W.F. (1999) A Joint Replenishment Policy for Multi-Echelon Inventory Control. International Journal of Production Economics, 59, 243-250. https://doi.org/10.1016/S0925-5273(98)00018-8

[28] Axsäter, S. and Juntti, L. (1996) Comparison of Echelon Stock and Installation Stock 
Policies for Two-Level Inventory Systems. International Journal of Production Economics, 45, 303-310. https://doi.org/10.1016/0925-5273(95)00121-2

[29] Paterson, C., Kiesmüller, G., Teunter, R., et al. (2011) Inventory Models with Lateral Transshipments: A Review. European Journal of Operational Research, 210, 125-136. https://doi.org/10.1016/j.ejor.2010.05.048

[30] Liao, Y., Shen, W., Hu, X., et al. (2014) Optimal Responses to Stockouts: Lateral Transshipment versus Emergency Order Policies. Omega, 49, 79-92. https://doi.org/10.1016/j.omega.2014.05.010

[31] Dan, B., He, Q.R. and Li, Y.Y. (2017) Decision-Making Model of Preventive Lateral Transshipment and Ordering for Perishable Product in Multi Periods. Journal of Industrial Engineering and Engineering Management, 31, 133-141. 